\title{
CALIDAD POSTCOSECHA DE FRUTOS DE PITAHAYA (Hylocereus undatus Haw.) COSECHADOS EN TRES ESTADOS DE MADUREZ
}

\author{
POSTHARVEST QUALITY OF PITAHAYA (Hylocereus undatus Haw.) \\ FRUITS HARVESTED IN THREE MATURITY STAGES
}

\section{Tomás Osuna Enciso ${ }^{1 *}$, Ma. Emilia Ibarra Zazueta ${ }^{1}$, Ma. Dolores Muy Rangel ${ }^{1}, J_{\text {J. Benigno Valdez Torres }}{ }^{1}$, Manuel Villarreal Romero ${ }^{2}$ y Sergio Hernández Verdugo ${ }^{2}$}

\footnotetext{
${ }^{1}$ Centro de Investigación en Alimentación y Desarrollo, A. C.-Unidad Culiacán. Carretera a Eldorado Km 5.5. 80110, Culiacán, Sinaloa, México. Tel/Fax: (667)7605536. ${ }^{2}$ Facultad de Agronomía, Universidad Autónoma de Sinaloa. Carretera a Eldorado km. 17.5. Culiacán, Sinaloa, México.

*Autor para correspondencia (tosuna@ciad.edu.mx)
}

\section{RESUMEN}

En este estudio se evaluó la calidad postcosecha de frutos de pitahaya (Hylocereus undatus Haw.) cosechados en tres estados de madurez: inicial, medio y completo, con 25-50, 50-75 y 75-100\% de color rojo en la cáscara, respectivamente. Los frutos fueron almacenados a $20 \pm 2{ }^{\circ} \mathrm{C}$. Se evaluaron características físicas $y$ químicas durante $12 \mathrm{~d}$ y las fisiológicas por $8 \mathrm{~d}$. La pérdida de peso al concluir el almacenamiento fue mayor $(P \leq 0.05)$ en frutos de madurez inicial (7.8 \%) que con madurez media $(6.1 \%)$ y completa (5.6 \%). El color de la cáscara varió $(P \leq 0.05)$ durante el almacenamiento y entre estados de madurez. Frutos con madurez media y completa concluyeron con valores de ángulo de matiz de 15.6 (rojo) y 8.6 (rojo morado), respectivamente, y los de madurez inicial con 31.7 (rojo-naranja). Firmeza, acidez titulable (AT), sólidos solubles totales (SST) y vitamina $\mathrm{C}$, disminuyeron $(\mathrm{P} \leq \mathbf{0 . 0 5})$ durante el almacenamiento. La firmeza se mantuvo más alta en frutos de madurez inicial. La AT disminuyó alrededor de $80 \%$ en los frutos de los tres estados de madurez, pero fue más alta en los cosechados con madurez inicial. Los SST tuvieron un descenso significativo $(P \leq \mathbf{0 . 0 5})$ con valores ${ }^{\circ}$ Brix más altos en frutos con madurez media y completa que en madurez inicial. La relación ${ }^{\circ}$ Brix/acidez (RBA) fue similar en los primeros 4 d de almacenamiento, pero los frutos en madurez inicial mantuvieron por más tiempo $(10$ d) los valores óptimos de RBA $(<40)$. La disminución de vitamina $\mathrm{C}$ fue mayor $(\mathrm{P} \leq \mathbf{0 . 0 5})$ en frutos con madurez inicial, mientras que la respiración fue mayor en frutos con madurez completa que con madurez inicial y media, y se observó un comportamiento no climatérico. La vida postcosecha para frutos con madurez media y completa fue entre 6 y 8 d, mientras que para madurez inicial fue $10 \mathrm{~d}$, pero en estos últimos el color rojo menos intenso afectó la calidad.

Palabras clave: Hylocereus undatus, calidad postcosecha, estados de madurez, respiración, vida de anaquel.

\section{SUMMARY}

In this study we evaluated the postharvest quality of pitahaya fruits (Hylocereus undatus Haw.) in three maturity stages: initial, medium, and complete, which corresponded to 25-50, 50-75, and 75-100 \% of red peel color, respectively. The fruits were stored under marketing conditions $\left(20 \pm 2{ }^{\circ} \mathrm{C}\right)$. Physical and chemical characteristics were evaluated during $12 \mathrm{~d}$ and the physiological characteristics during $8 \mathrm{~d}$. By the end of the storage period, weight loss was higher $(P \leq 0.05)$ in fruits harvested at initial maturity $(7.8$ $\%)$, than in fruits of medium $(6.1 \%)$ and complete $(5.6 \%)$ maturities. Peel color was different $(P \leq 0.05)$ between maturities and storage times. At day 12, fruits harvested at medium and complete maturities showed hue angle $\left({ }^{\circ} \mathrm{Hue}\right)$ values of 15.6 (red) and 8.6 (red-dwelled), respectively, whereas fruits harvested at initial maturity ended with 31.7 (red-orange). Firmness, titratable acidity (TA), total soluble solids (TSS) and vitamin $\mathrm{C}$ decreased (P $\leq$ $0.05)$ during storage. Firmness remained higher $(P \leq 0.05)$ in fruits harvested initial maturity. TA decreased $80 \%(P \leq 0.05)$ in fruits of the three maturity stages, but it remained higher in fruit of the initial maturity stage. TSS had significant reduction $(P \leq 0.05)$ during storage, and ${ }^{\circ}$ Brix values were higher $(P \leq 0.05)$ in fruits harvested in medium and complete maturity stages, as compared with fruits harvested at initial maturity. The Brix/acidity ratio (BAR) was similar in the first $4 \mathbf{d}$ of storage, but the fruits in initial maturity showed longer $(10 \mathrm{~d} ; \mathrm{P} \leq \mathbf{0 . 0 5})$ optimal values of BAR $(<40)$. Decrease in vitamin $C$ was higher $(P \leq 0.05)$ in fruit harvested in complete maturity. Respiration rate was higher $(\mathbf{P} \leq$ 0.05) in fruits with complete maturity than in fruits with initial maturity and medium maturity, and a non-climacteric behavior was observed. Shelf life for fruits with medium and complete maturity was between 6 and $8 \mathrm{~d}$, whereas for initial maturity was $10 \mathrm{~d}$, but in the last ones the less intense red color affected fruit quality.

index words: Hylocereus undatus, postharvest quality, maturity stages, respiration, shelf life. 


\section{INTRODUCCIÓN}

La pitahaya (Hylocereus undatus Haw.) es una cactácea nativa de América, cuya adaptabilidad a diversas condiciones ambientales ha favorecido su introducción a países con marcadas diferencias en clima y suelo (Mizrahi et al., 2002). Se distribuyó de América a Asia (China, Vietnam, Indonesia y Corea) y Medio Oriente (Israel), donde ha tenido amplia aceptación. Nicaragua, Colombia y México son los países de América que cuentan con plantaciones comerciales (Meraz et al., 2003). En México, el cultivo sobresale en los Estados de Yucatán, Quintana Roo y Puebla, con 328.8, 143.0 y 15.5 ha, respectivamente (SAGARPA, 2009). En el Estado de Sinaloa, México, se inició recientemente el cultivo de pitahaya (1.0 ha) y ya se han obtenido las primeras cosechas, cuyos frutos se venden en la región; a mediano plazo se pretende incursionar en el mercado de EE. UU., por lo que se requiere conocer las prácticas convenientes de cosecha y manejo postcosecha (Osuna, 2006).

Según Corrales (2003), se sabe poco de los índices de cosecha y del comportamiento postcosecha del fruto de pitahaya, así como de los parámetros de calidad de las diferentes especies cultivadas, y no se ha abordado el problema de la alta perecibilidad. Estudios realizados en Israel (Nerd et al., 1999) y Vietnam (To et al., 2002) mostraron que la cosecha del fruto de Hylocereus undatus se efectúa cuando éstos adquieren el color rojo, que ocurre entre los 28 y 30 d después de antesis. Resultados similares fueron obtenidos por Centurión et al. (2008), quienes en un estudio sensorial encontraron que los frutos más aceptados se cosecharon entre los 25 y 31 d después de antesis. Por su parte, Merten (2003) indicó que en California, EE.UU., la maduración de las pitahayas ocurre entre 40 y $45 \mathrm{~d}$ después de la floración, tiempo en que los frutos alcanzaron el nivel máximo de sólidos solubles totales, que va de 13 a $16{ }^{\circ}$ Brix.

En México, Centurión et al. (1999) cosecharon frutos a los 26 y 30 d después de la floración, con color verde claro y rosa, respectivamente y los conservaron a temperatura ambiente por $15 \mathrm{~d}$; los de color verde claro perdieron más peso (14\%) que los frutos cosechados en color rosa (5\%); además, al concluir el almacenamiento, los frutos verde claro presentaron color rojo menos intenso que los cosechados en color rosa. Nerd et al. (1999) reportaron que en frutos de $H$. undatus el inicio de color ocurrió entre los 24 y 25 d después de antesis, y que de 4 a 5 d después se tornan completamente rojos; estos autores concluyeron que el desarrollo del color de los frutos se relaciona con contenido de sólidos solubles totales. Resultados similares fueron presentados por Centurión et al. (2008), cuyos frutos cosechados $20 \mathrm{~d}$ después de antesis en color verde claro presentaron $4.6^{\circ} \mathrm{Brix}$, mientras que los cosechados $25 \mathrm{~d}$ después de antesis, en color rosa, registraron 9.5 ${ }^{\circ}$ Brix. En tanto, Merten (2003) indicó que los sólidos solubles totales de frutos de $H$. undatus al momento de cosecha fluctuaron entre 13 y $16{ }^{\circ}$ Brix.

Nerd et al. (1999) manifestaron que la acidez titulable de las pitahayas se reduce considerablemente durante el almacenamiento, ya que en un estudio con frutos de $H$. undatus y $H$. polyrhizus observaron que el descenso de la acidez titulable fue de $50 \%$ en cuatro semanas de almacena-miento a $20{ }^{\circ} \mathrm{C}$. Centurión et al. (1999) registraron $1.0 \%$ de ácido cítrico en frutos de $H$. undatus con cáscara de color verde claro al momento de cosecha, valor que descendió a $0.28 \%$ después de dos semanas de almacenamiento a temperatura ambiente. Por su parte, To et al. (2002) mostraron que la acidez titulable se mantuvo en $0.40 \%$ de ácido cítrico en frutos maduros, y propusieron que el mejor indicador de sabor en pitahaya es la relación ${ }^{\circ}$ Brix/acidez, cuyo valor ideal es de alrededor de 40 .

La firmeza en frutos de $H$. undatus se reduce considerablemente cuando alcanzan madurez completa; frutos con 6.3 newtons $(\mathrm{N})$ disminuyeron a $3.8 \mathrm{~N}$ durante $14 \mathrm{~d}$ de almacenamiento a $20{ }^{\circ} \mathrm{C}$ (To et al., 2002). Asimismo, Centurión et al. (2008) reportaron $6.3 \mathrm{~N}$ de firmeza en frutos cosechados $31 \mathrm{~d}$ después de antesis. Wall y Khan (2008) en Hawaii, cosecharon frutos maduros entre 45 y 50 d después de antesis con firmeza de $5.7 \mathrm{~N}$. La firmeza es una variable poco evaluada en los estudios postcosecha de pitahaya. Al respecto, Kader et al. (2002) señalaron que esta característica es determinante en la vida de anaquel de los frutos carnosos.

Centurión et al. (1999) refirieron que a temperatura ambiente la vida postcosecha de los frutos de pitahaya es de $8 \mathrm{~d}$ para los cosechados en color verde y $6 \mathrm{~d}$ para los cosechados en color rosa. Nerd et al. (1999) almacenaron frutos de $H$. undatus y $H$. polyrhizus a 14 y $20{ }^{\circ} \mathrm{C}$, y lograron conservar sus cualidades de comercialización por dos y una semanas, respectivamente. Rodríguez et al. (2005) establecieron que en pitahaya amarilla (Selenicereus megalanthus Haw.) los frutos almacenados a $8{ }^{\circ} \mathrm{C}$ tuvieron mayor vida poscosecha $(19$ d) que los almacenados a $19{ }^{\circ} \mathrm{C}(7 \mathrm{~d})$, aunque los autores observaron desarrollo de daños por frío (picaduras de color rojizo de 2 $\mathrm{mm}$ de diámetro) a partir del día 13 , en $5 \%$ de la superficie de la fruta. Balois-Morales et al. (2008) reportaron que el sistema antisenescencia regulado por catalasa (CAT) y superóxido dismutasa (SOD) se afectó parcialmente por el frío, ya que la actividad de CAT disminuyó a temperaturas bajas $\left(3.7\right.$ y $\left.11.0{ }^{\circ} \mathrm{C}\right)$ mientras 
que la SOD se mantuvo estable, con incremento importante a $11{ }^{\circ} \mathrm{C}$.

La respiración afecta la vida postcosecha de los frutos. En general, existe una relación inversa entre las tasas de respiración y la vida postcosecha de éstos, pues a mayor respiración son más perecederos (Kader y Saltveit, 2003). Nerd et al. (1999) midieron la producción de etileno y $\mathrm{CO}_{2}$ en frutos de $H$. undatus y $H$. polyrhizus durante $6 \mathrm{~d}$ a 20 ${ }^{\circ} \mathrm{C}$ y encontraron que tienen un comportamiento no climatérico; en contraste, Rodríguez et al. (2005) señalaron que la pitahaya amarilla (Selenicereus megalanthus Haw.) tiene un comportamiento climatérico.

La información generada en postcosecha de $H$. undatus (Centurión et al., 1999; Nerd et al., 1999; To et al., 2002; Centurión et al., 2008), podría ser insuficiente para prescribir la calidad y vida de anaquel del fruto cosechado con base al color de cáscara. Si bien se recomienda cosechar cuando los frutos están completamente rojos, la vida útil de éstos es muy corta. Nerd et al. (1999) recomiendan explorar la cosecha de pitahaya en etapa inicial de maduración, que ellos llaman "primer color", con el fin de ampliar la vida postcosecha. Lo anterior motivó la realización del presente estudio que tuvo como objetivo establecer criterios para llevar a cabo la cosecha de frutos de pitahaya ( $H$. undatus) y determinar la calidad y vida postcosecha en tres estados de madurez, definidos mediante una escala (\%) de color rojo de la superficie de la cáscara, y almacenados en condiciones de mercadeo (20 $\left.\pm 2{ }^{\circ} \mathrm{C}\right)$.

\section{MATERIALES Y MÉTODOS}

Frutos de pitahaya pulpa blanca y cáscara roja se cosecharon en un huerto de cuatro años, plantado con un clon introducido de Puebla, México. Dicho huerto se ubica en la sindicatura de Costa Rica ( $24^{\circ} 34^{\prime}$ 56' ' LN y $107^{\circ} 16^{\prime}$ 51' LO), en Culiacán, Sinaloa, México. Los frutos fueron clasificados de acuerdo con una escala subjetiva de color rojo de la superficie de la cáscara; 25 a 50,50 a 75 y 75 a $100 \%$, que correspondió a tres estados de madurez: inicial, media y completa, respectivamente (Figura 1); en la clasificación participó sólo una persona. Los frutos pesaban de $450 \mathrm{~g}$ en promedio, y sus diámetros ecuatorial y polar eran de 8.1 y $13.6 \mathrm{~cm}$, respectivamente. Las pitahayas se almacenaron en cajas de plástico en simulación de mercadeo $\left(20 \pm 2{ }^{\circ} \mathrm{C}\right.$ y $85-90 \%$ HR). Se evaluó diariamente pérdida de peso, color externo y producción de $\mathrm{CO}_{2}$, y cada 2 d se midió firmeza, acidez titulable (AT) y sólidos solubles totales (SST); con los valores de las dos variables anteriores se obtuvo la relación ${ }^{\circ}$ Brix/acidez (RBA). Asimismo, se evaluó contenido de vitamina $\mathrm{C}$ al inicio $\mathrm{y}$ al final del almacenamiento (0 y $12 \mathrm{~d})$.

\section{Análisis físicos}

Se determinó pérdida de peso en una muestra de 10 frutos por cada estado de madurez. El peso se registró en una balanza digital Sartorius AND GF-2000®. EI porcentaje de pérdida de peso diario acumulado se determinó con la metodología propuesta por Díaz-Pérez (1998). En el mismo grupo de frutos se midió el color externo en tres puntos de la parte central del fruto, y también midieron los valores de $\mathrm{L}^{*}, \mathrm{a}^{*} \mathrm{y} \mathrm{b}^{*}$ con un colorímetro Minolta CR-300® (New Jersey, USA); los resultados se expresaron en ángulo de matiz ( $\left.{ }^{\circ} \mathrm{Hue}\right)$ (Minolta, 1994). La firmeza se midió en la pulpa de cinco frutos con un penetrómetro digital Chatillon TCD $200 \AA$, equipado con una sonda cilíndrica de punta plana de 8 $\mathrm{mm}$ de diámetro con penetración de $1.5 \mathrm{~cm}$; se hicieron tres lecturas sobre el diámetro ecuatorial del fruto y los valores se registraron en newtons (Bourne, 1980).

\section{Análisis químicos}

El análisis de acidez titulable (AT) y sólidos solubles totales (SST) se efectuó con la metodología de la AOAC (1998). Se tomó una muestra de cinco frutos por estado de madurez y de cada fruto se licuaron $10 \mathrm{~g}$ de pulpa en $50 \mathrm{~mL}$ de agua destilada; la mezcla se filtró en tela de organza y se tomaron $50 \mathrm{~mL}$ para medir acidez titulable con un titulador Mettler Toledo Mod. DL-50®, los resultados se expresaron en porciento de ácido málico. Del filtrado se tomó una muestra para determinar el contenido de sólidos solubles totales en un refractómetro Mettler Toledo Mod. RE40D®, y sus resultados se expresaron en ${ }^{\circ}$ Brix considerando la dilución $(50 \mathrm{~mL})$. Con ambas variables se calculó la relación ${ }^{\circ} \mathrm{Brix} / \mathrm{acidez}$ (RBA). La vitamina $\mathrm{C}$ se analizó con la metodología de Gökmen et al. (2000); se preparó un extracto con $10 \mathrm{~g}$ de muestra y $40 \mathrm{~mL}$ de agua destilada, del cual se tomó 1 $\mathrm{mL}$ y se le adicionó $1 \mathrm{mg}$ de dithiothreitol (agente reductor) para cuantificar vitamina $\mathrm{C}$ total; posteriormente se inyectaron $20 \mu \mathrm{L}$ en un cromatógrafo de líquidos Varian® equipado con un detector UV con arreglo de diodos ProStar 330, una bomba terciaria ProStar 230, columna Varian C18 de 150 x $4.60 \mathrm{~mm}$; el cromatógrafo fue operado a temperatura ambiente a una longitud de onda de $254 \mathrm{~nm}$. Se usó fase móvil de $\mathrm{KH}_{2} \mathrm{PO}_{4} 0.2 \mathrm{M}$ y un flujo de $0.5 \mathrm{~mL} \mathrm{~min}{ }^{-1}$. Para el análisis se preparó una curva estándar de ácido ascórbico (SIGMA $®$ ) en concentraciones de $0,10,20,30$ y $40 \mathrm{mg}$ $\mathrm{L}^{-1}$. 


\section{Análisis fisiológico}

Se midió la producción de bióxido de carbono $\left(\mathrm{CO}_{2}\right)$ de un fruto (unidad experimental), con tres réplicas por cada estado de madurez. Cada fruto se pesó y se colocó en un frasco de vidrio herméticamente cerrado, abastecido de un flujo de aire continuo $\left(60 \mathrm{~mL} \mathrm{~min}{ }^{-1}\right)$. Diariamente se tomó una muestra de la salida de aire para cuantificar la tasa respiratoria. Las muestras se inyectaron en un cromatógrafo de gases Varian Modelo $3300 \AA$, equipado con un detector de conductividad térmica operado a una temperatura inicial de $70{ }^{\circ} \mathrm{C}$ con incremento hasta 120 ${ }^{\circ} \mathrm{C}$; columna HayeSepQ 100/120 de $3 \mathrm{~mm}$ de diámetro y $180 \mathrm{~cm}$ de largo, con helio como gas acarreador. La corrida se hizo con tiempos de retención de $1.06 \mathrm{~min}, \mathrm{y}$ los resultados se obtuvieron al cuantificar el área bajo la curva de $\mathrm{CO}_{2}$, mediante la integración de una curva estándar de calibración con $0.2,0.4,0.6,0.8$ y $1.0 \mathrm{~mL}$ de $\mathrm{CO}_{2}(0.52 \%$, $\mathrm{AGA} \circledast$, Sinaloa, México). Los resultados se reportaron en $\mathrm{mg} \mathrm{CO} \mathrm{kg}^{-1} \mathrm{~h}^{-1}$ (Báez et al., 1997).

\section{Análisis estadístico}

Las variables pérdida de peso, color y producción de $\mathrm{CO}_{2}$ fueron analizadas mediante un diseño de medidas repetidas con dos factores, madurez (inicial, media y completa) y tiempo (días de evaluación). Las variables firmeza, acidez titulable, SST y vitamina $\mathrm{C}$ se analizaron mediante un diseño totalmente al azar de dos factores: estado de madurez (inicial, media y completa) y tiempo (días de evaluación). Para cada variable se hizo un análisis de varianza, y en caso de efectos significativos se determinaron las diferencias de tratamientos mediante la prueba de Tukey $(\mathrm{P} \leq 0.05)$, con el programa estadístico computacional MINITAB (2004) versión 14.0.

\section{RESULTADOS Y DISCUSIÓN}

\section{Análisis físicos}

Pérdida de peso. La pérdida de peso acumulada presentó cambios significativos $(\mathrm{P} \leq 0.05)$ durante el tiempo de almacenamiento y entre estados de madurez. Los frutos cosechados en madurez inicial tuvieron la mayor pérdida de peso después de $12 \mathrm{~d}(7.8 \%)$, mientras que los cosechados con madurez media y completa registraron 6.1 y $5.6 \%$, respectivamente, sin diferencia entre éstos (Figura 2). Centurión et al. (1999) también encontraron pérdidas de peso de 14 y $5 \%$ en frutos cosechados al inicio de su madurez (cáscara color verde claro) y completamente maduros (cáscara color rojo), respectivamente, y almacenados a temperatura ambiente durante $11 \mathrm{~d}$.
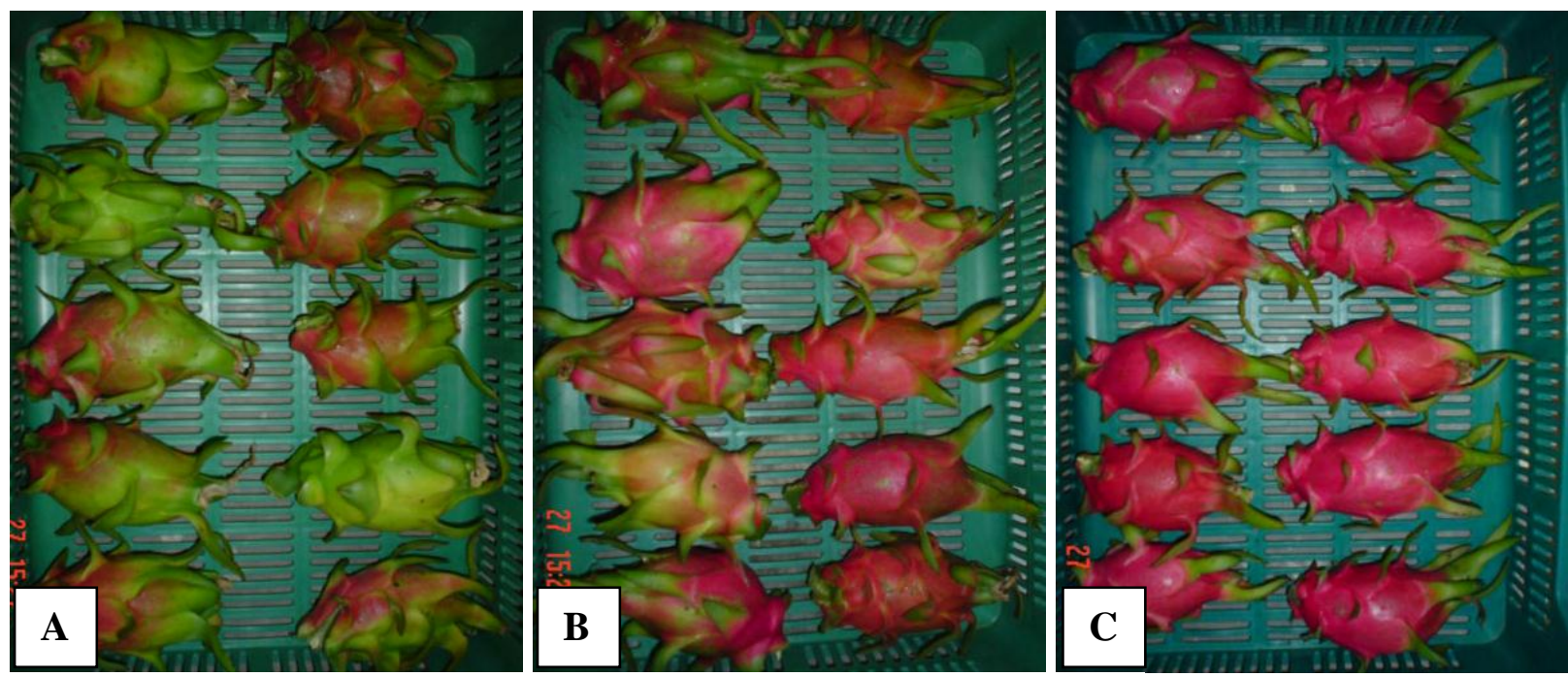

Figura 1. Frutos de pitahaya cosechados en madurez inicial (A), media (B) y completa (C). 
De acuerdo con Wills et al. (1998), una pérdida de peso superior a $5 \%$ puede ser suficiente para disminuir la calidad en diferentes frutos. En el presente estudio se observó que con $5 \%$ de pérdida de peso, las pitahayas presentaron marchitez ligera y muerte de tejido en el borde de las brácteas, lo cual ocurrió en el estado de madurez inicial después de $7 \mathrm{~d}$ de almacenamiento, mientras que en los estados de madurez medio y completo ocurrió a los 10 y 11 d, respectivamente (Figura 3).
Con excepción de la marchitez de brácteas, los frutos de los tres estados de madurez no mostraron deshidratación en el resto de su superficie. La mayor pérdida de peso ocurrida en los frutos cosechados en madurez inicial puede estar asociada con menor deposición de cera en la epidermis. Teixeira et al. (2005), en un estudio con Platonia insignis, concluyeron que la mayor pérdida de peso en frutos cosechados inmaduros se debió a que poseen cutícula más delgada que los frutos cosechados maduros.

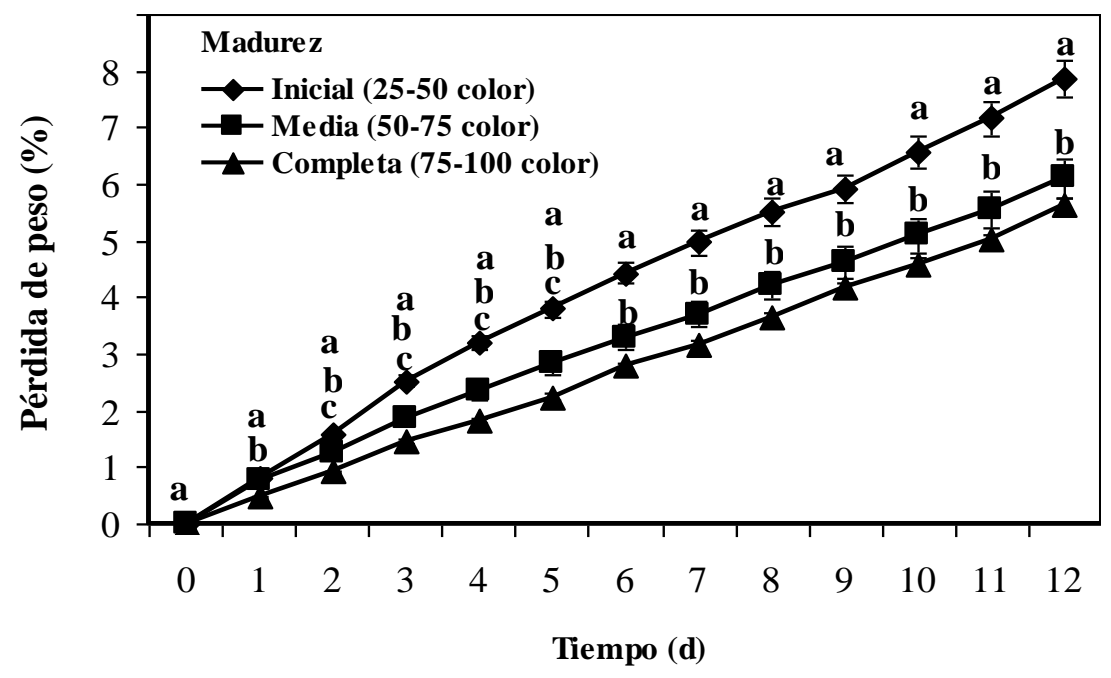

Figura 2. Porcentaje de pérdida de peso acumulada en frutos de pitahaya cosechados en tres estados de madurez y almacenados a $20 \pm 2{ }^{\circ} \mathrm{C}$. Medias con la misma letra en el día de muestreo no son estadísticamente diferentes. Barras verticales indican el error estándar $(\mathbf{n}=10)$.

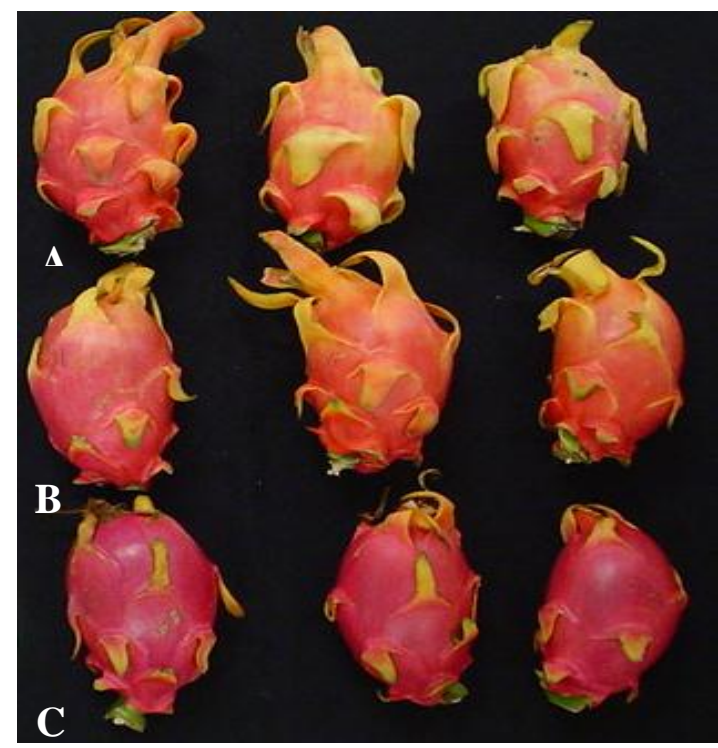

Figura 3. Frutos de pitahaya cosechados en madurez inicial (A), media (B) y completa (C), después de $12 \mathrm{~d}$ de almacenamiento a $20 \pm 2{ }^{\circ} \mathrm{C}$.
Color de cáscara. El color de cáscara, medido como ángulo de matiz $\left({ }^{\circ} \mathrm{Hue}\right)$, presentó cambios $(\mathrm{P} \leq 0.05)$ durante el tiempo de almacenamiento de los frutos cosechados en madurez inicial y media, mientras que en los cosechados con madurez completa se mantuvo sin cambios. Asimismo, esta variable fue diferente $(\mathrm{P} \leq 0.05)$ entre estados de madurez (Figura 4). Los valores ${ }^{\circ} \mathrm{Hue}$ obtenidos en el día cero fueron 115.0, 75.0 y 17.2 para madurez inicial, media y completa, respectivamente, los cuales corresponden a los colores verde, amarillo-rosado y rojo, respectivamente. Después de $12 \mathrm{~d}$ el ángulo de matiz de los frutos cosechados en los estados de madurez inicial, media y completa, fue de 31.8, 15.6 y 8.3, respec-tivamente, valores que indican colores rojonaranja, rojo y rojo-púrpura, respectivamente (Figura 3).

Según Le Bellec et al. (2006), el color rojo de la piel y del fruto de Hylocereus se debe a pigmentos de betalainas. Phebe et al. (2009) encontraron una correlación significativa negativa entre valores de ${ }^{\circ} \mathrm{Hue}$ y el contenido total de betacianinas de cáscara y fruto de $H$. 
polyrhizus, con un drástico incremento de 90 y $65 \%$, respectivamente, entre los 25 y los 30 d después de antesis. To et al. (2002) señalaron que se requieren valores menores o iguales a $30{ }^{\circ} \mathrm{Hue}$ para que las pitahayas se comercialicen. En el presente estudio los frutos con madurez completa registraron valores inferiores a $30{ }^{\circ}$ Hue desde el inicio de almacenamiento, los de madurez intermedia tuvieron este valor al tercer día, mientras que los frutos de madurez inicial no alcanzaron este nivel durante el almacenamiento, lo que afectó su calidad (Figura 4).



Figura 4. Color externo $\left({ }^{\circ} \mathrm{Hue}\right)$ en frutos de pitahaya cosechados en tres estados de madurez y almacenados a $20 \pm 2{ }^{\circ} \mathrm{C}$. Medias con la misma letra en el día de muestreo no son estadísticamente diferentes. Barras verticales indican el error estándar $(n=10)$.

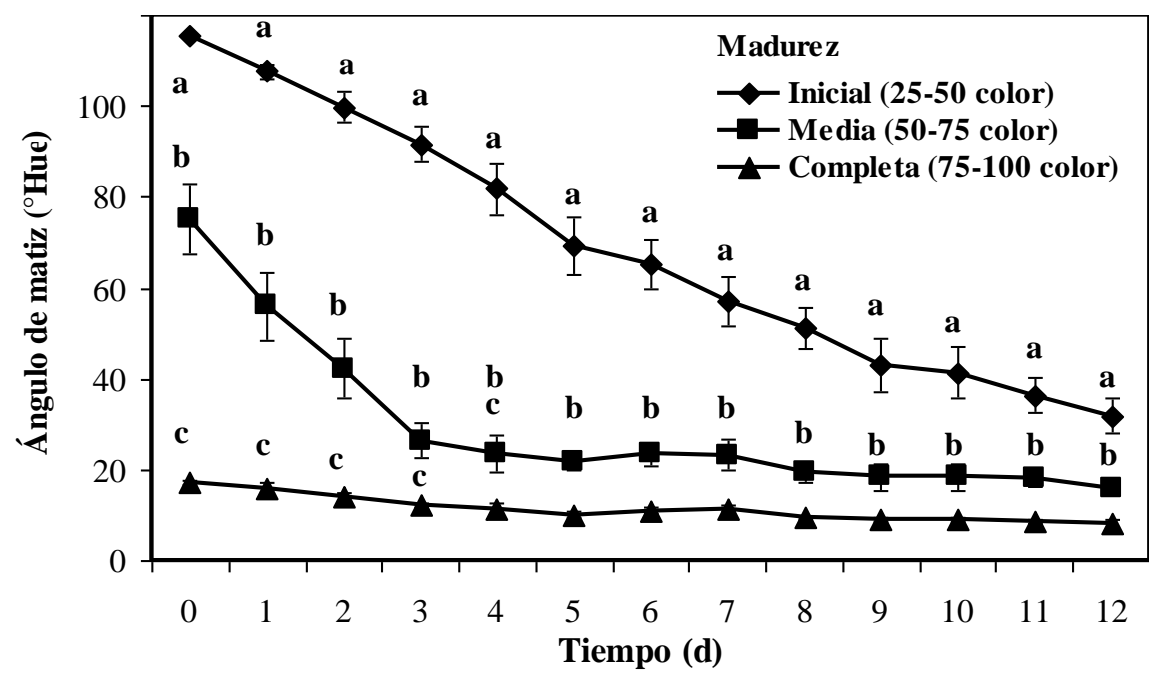

Figura 5. Firmeza en frutos de pitahaya cosechados en tres estados de madurez y almacenados a $20 \pm 2{ }^{\circ} \mathrm{C}$. Medias con la misma letra en el día de muestreo no son estadísticamente diferentes. Barras verticales indican el error estándar $(\mathbf{n}=\mathbf{5})$.

Cuadro 1. Concentración de vitamina $\mathrm{C}$ en frutos de pitahaya cosechados en tres estados de madurez y almacenados a $20 \pm 2{ }^{\circ} \mathrm{C}$.

\begin{tabular}{cccc}
\hline \multirow{3}{*}{$\begin{array}{c}\text { Almacenamiento } \\
\text { en días }\end{array}$} & Inicial $(25-50 \%$ color $)$ & Media $(50-75 \%$ color $)$ & Completa (75-100 \% color) \\
\cline { 2 - 4 } & & Vitamina C $(\mathrm{mg} \mathrm{100} \mathrm{g}$ $) \pm$ ee & $7.93 \pm 0.38 \mathrm{~b}$ \\
\cline { 2 - 4 } 12 & $9.31 \pm 0.36 \mathrm{a}$ & $8.48 \pm 0.18 \mathrm{ab}$ & $7.24 \pm 0.19 \mathrm{~b}$ \\
\hline
\end{tabular}

Medias con la misma letra en la misma hilera no son estadísticamente diferentes (Tukey, 0.05); ee = error estándar $(\mathrm{n}=3)$. 
Firmeza. La firmeza de los frutos disminuyó $(\mathrm{P} \leq 0.05)$ en los primeros $6 \mathrm{~d}$ de almacenamiento, y fue mayor en frutos cosechados en madurez inicial que en frutos con madurez media y completa cuyo firmeza fue similar (Figura 5). Entre los 0 y 6 d la firmeza de los frutos cosechados en madurez inicial, media y completa pasó de 7.1 a 5.0 , de 5.4 a 4.5 y de 6.0 a $4.2 \mathrm{~N}$, respectivamente, pero después de este periodo la firmeza no se modificó. Este comportamiento de la firmeza también fue reportado por To et al. (2002) en frutos de $H$. undatus almacenados a $20{ }^{\circ} \mathrm{C}$ durante $14 \mathrm{~d}$, los cuales iniciaron en $6.3 \mathrm{~N}$ y concluyeron con $3.8 \mathrm{~N}$. Centurión et al. (1999) indicaron que el ablandamiento de la pulpa de pitahaya se relaciona con un incremento en la actividad de la enzima pectinmetilesterasa durante la maduración. Asimismo, Kader et al. (2002) señalaron que el ablandamiento de los frutos carnosos durante su madurez, se debe a la disolución de la lámina media de sus tejidos.

\section{Análisis químicos}

Acidez titulable (AT). La acidez titulable de las pitahayas, medida como porciento de ácido málico, disminuyó en $80 \%$ durante el de almacenamiento (Figura 6A). Los frutos de madurez media y completa tuvieron los valores más bajos de AT, y fueron significativamente diferentes a los cosechados con madurez inicial que conservaron valores de AT más altos $(\mathrm{P} \leq 0.05)$. El porciento de ácido málico de los frutos cosechados en madurez inicial, media y completa fue de $0.92,0.76 \mathrm{y}$ 0.63 al inicio del almacenamiento, y concluyeron con valores de $0.18,0.14$ y 0.10 , respectivamente (Figura 6A). En frutos de $H$. polyrhizus y $H$. undatus Nerd et al. (1999) encontraron una tendencia similar de la AT, la cual fue mayor en frutos en color aún cambiante que en frutos de color avanzado; además, ambos grupos registraron $50 \%$ de descenso en la AT durante las cuatro semanas de almacenamiento, $30 \%$ menos que los frutos del presente estudio. Wills et al. (1998) señalaron que la disminución de los ácidos orgánicos durante la maduración de los frutos se debe a que son utilizados como sustrato en la respiración. Según Centurión et al. (1999), se requiere un valor de $0.24 \%$ de AT para que el fruto mantenga un buen sabor. En el presente trabajo, este valor se observó entre los 10,8 y 6 d para madurez inicial, media y completa, respectivamente (Figura 6A).

Sólidos solubles totales (SST). Los SST descendieron $(\mathrm{P} \leq 0.05)$ durante los primeros $4 \mathrm{~d}$ de almacenamiento de los frutos (Figura 6B). Los frutos de madurez completa y media tuvieron valores de ${ }^{\circ}$ Brix más altos que los frutos en madurez inicial, pero sin diferencia significativa entre tratamientos. Al inicio y conclusión del almacenamiento, la concentración de SST para frutos cosechados en madurez completa, media e inicial estuvo en los rangos 13.6-11.6, 12.4-11.3 y 12.2-11.2 ${ }^{\circ}$ Brix, respectivamente (Figura 6B). Nerd et al. (1999) manifestaron que durante el almacenamiento no se incrementó la concentración de SST en las pitahayas, porque acumulan la mayor cantidad de azúcares en la fase final de desarrollo en la planta, que coincide con el cambio de color de la cáscara. Asimismo, Centurión et al. (2008) reportaron una estrecha relación entre el desarrollo de color y el incremento de SST, donde frutos con $20 \mathrm{~d}$ de desarrollo registraron $4.6{ }^{\circ}$ Brix mientras que con $31 \mathrm{~d}$ alcanzaron $12.6^{\circ}$ Brix. En el presente estudio, los frutos con madurez media y completa tuvieron los valores más altos de SST, que corresponden con los valores más bajos de ${ }^{\circ} \mathrm{Hue}$.

Relación Brix/acidez (RBA). En los primeros $4 \mathrm{~d}$ de almacenamiento la RBA de los tres estados de madurez, osciló con valores entre 13 y 22 (Figura 6C); posteriormente, los valores se incrementaron $(\mathrm{P} \leq 0.05)$. Esta variable fue similar en frutos de madurez media y completa al inicio, pero al concluir el almacenamiento sus valores fueron de 82.1 y 95.6 , respectivamente, que son superiores $(\mathrm{P} \leq 0.05)$ a los de frutos en madurez inicial, cuyo valor fue de 60 . El aumento en la RBA se debió a la caída drástica de la acidez titulable (Figura 6A), por lo que en estos frutos un valor alto en la RBA no es un indicador de calidad, ya que los SST no se incrementan. Esta relación indica un sabor "insípido" a los frutos de pitahaya, característica reportada por Centurión et al. (1999) y Nerd et al. (1999). El valor ideal de RBA para el consumo de frutos de $H$. undatus es menor a 40 (Nerd et al., 1999; To et al., 2002). De acuerdo con este valor, las pitahayas estudiadas tuvieron un rango aceptable de $6 \mathrm{~d}$ para los frutos cosechados en madurez completa, de entre 6 y $8 \mathrm{~d}$ para los de madurez media, y de $10 \mathrm{~d}$ para los de madurez inicial.

Vitamina C. Esta variable, que se relaciona con la calidad de los frutos, disminuyó $(\mathrm{P} \leq 0.05)$ durante el almacenamiento de las pitahayas (Cuadro 1). El contenido de vitamina $\mathrm{C}$ fue mayor $(\mathrm{P} \leq 0.05)$ en los frutos cosechados en madurez inicial, y no se detectó diferencia entre los frutos cosechados con madurez media y completa. Según Rodríguez et al. (2005), la disminución de los ácidos orgánicos en los frutos de pitahaya se debe a que son usados como sustrato en la respiración durante el almacenamiento. Centurión et al. (2008) registraron descenso de vitamina $\mathrm{C}$ durante la maduración del fruto de $H$. undatus; cuando se cosechó $20 \mathrm{~d}$ después de floración alcanzó $14.7 \mathrm{mg} 100 \mathrm{~g}^{-1}$ y cuando cumplió $31 \mathrm{~d}$ descendió a $9.6 \mathrm{mg} 100 \mathrm{~g}^{-1}$. Los ácidos málico y cítrico 
también disminuyen durante la maduración del fruto, ya que se usan para la síntesis de nuevos compuestos que dan sabor y aroma (Ladaniya, 2008).

\section{Análisis fisiológico}

Los frutos cosechados en madurez completa tuvieron una tasa respiratoria significativa más alta $(\mathrm{P} \leq 0.05)$ que los cosechados en madurez inicial y media (Figura 7); sin embargo, por fecha de muestreo la respiración no difirió entre estados de madurez. En los de madurez completa la producción de $\mathrm{CO}_{2}$ fluctuó de 45 a $55 \mathrm{mg} \mathrm{CO} \mathrm{kg}^{-1} \mathrm{~h}^{-1}$, mientras que en los frutos con madurez inicial y media osciló de 41 a 43 y 40 a 44 mg CO $\mathrm{kg}^{-1} \mathrm{~h}^{-1}$, respectivamente; la mayor respiración en frutos con madurez completa concuerda con las afirmaciones de


Higura o. Evolucion de componentes quimicos de frutos de pitahaya cosechados en tres estados de madurez y almacenados a $20 \pm 2{ }^{\circ} \mathrm{C}$. AT $=$ acidez titulable; SST sólidos solubles totales. Medias con la misma letra en el día de muestreo no son estadísticamente diferentes. Barras verticales indican el error estándar $(\mathbf{n}=10)$. 
Kader y Saltveit (2003), quienes comentan que los frutos carnosos liberan etileno al llegar a madurez, con un aumento proporcional en la respiración para atender las necesidades energéticas de las enzimas que contribuyen a su senescencia. Por su parte, Paliyath y Murr (2008) indicaron que en frutos no climatéricos el incremento en respiración que ocurre, en respuesta a la síntesis de etileno, no acelera el proceso de maduración como ocurre en los frutos climatéricos. De acuerdo con el comportamiento fisiológico de los frutos estudiados, se coincide con la opinión de Nerd et al. (1999) y To et al. (2002), quienes reportaron un comportamiento no climatérico en frutos del género Hylocereus.

En los frutos de maduración completa la vida de anaquel se interrumpió al ser atacados por hongos de los géneros Aspergillus, Colletotrichum y Fusarium, lo que obligó a interrumpir el muestreo de $\mathrm{CO}_{2}$ al día 6 (Figura 7).

\section{CONCLUSIONES}

Los frutos de pitahaya cosechados en madurez media y completa mantuvieron mejores características de color de cáscara y nivel de sólidos solubles totales durante $12 \mathrm{~d}$ de almacenamiento $\left(20 \pm 2{ }^{\circ} \mathrm{C}\right)$, que los frutos cosechados en madurez inicial. Sin embargo, la rápida disminución de la acidez afectó su calidad, que se mantuvo en condiciones aceptables entre 6 y $8 \mathrm{~d}$. Por otra parte, los frutos cosechados en madurez inicial conservaron los mayores niveles de firmeza, acidez y vitamina $\mathrm{C}$ y mejor relación ${ }^{\circ}$ Brix/acidez, características que mantuvieron hasta el día 10 de almacenamiento; en estos frutos, el color rojo de la cáscara fue menos intenso que en frutos cosechados en madurez media y completa, lo que redujo su calidad.

\section{AGRADECIMIENTOS}

A la Fundación Produce Sinaloa, A. C., Zona Centro y el Consejo Estatal de Ciencia y Tecnología, por el apoyo financiero. A la M. C. Laura Aracely Contreras Angulo, M. C. Rosabel Vélez de la Rocha y Rosalba Contreras Martínez, por su colaboración en los trabajos de laboratorio.

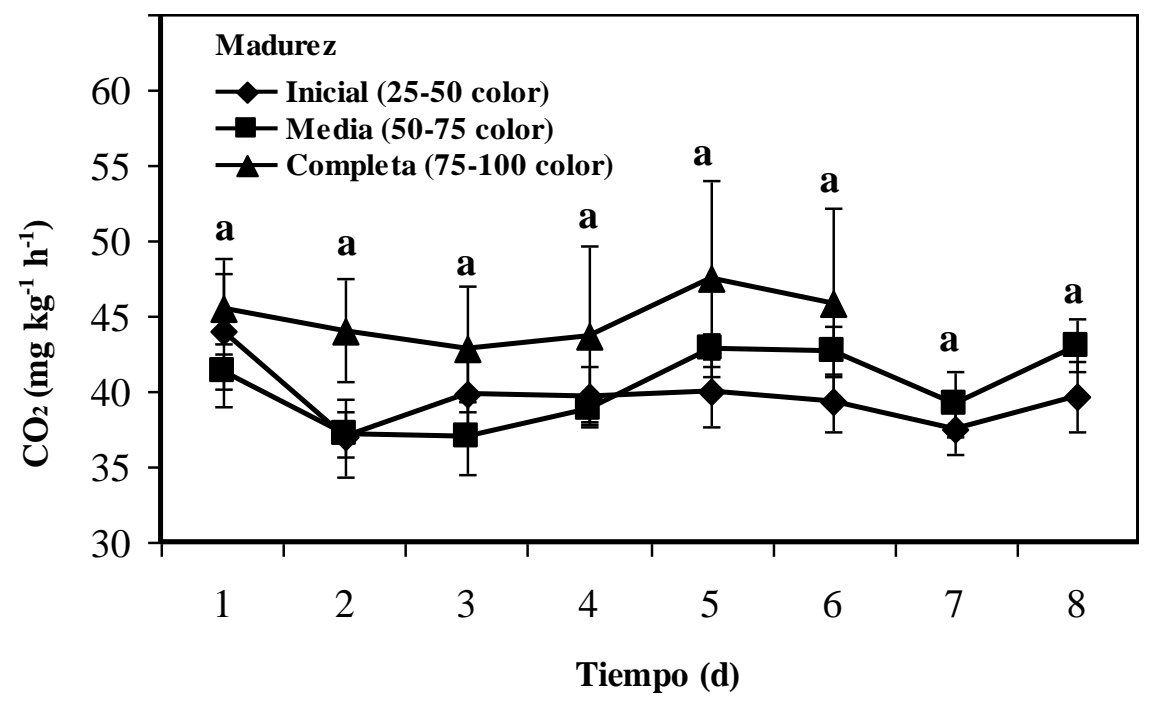

Figura 7. Producción de $\mathrm{CO}_{2}$ en frutos de pitahaya cosechados en tres estados de madurez y almacenados a $20 \pm 2{ }^{\circ} \mathrm{C}$. Medias con la misma letra en el día de muestreo no son estadísticamente diferentes. Barras verticales indican el error estándar $(n=3)$. 


\section{BIBLIOGRAFÍA}

AOAC, Association of Official Analytical Chemists (1998) Official Methods of Analysis. 16th ed. S William (ed). Published by the Association of Official Analytical Chemists. Washington, D.C. USA. CD-Rom.

Báez M A, J Siller, J B Heredia, E Araiza, R S García, M D Muy (1997) Fisiología poscosecha de frutos de chicozapote (Achras sapota L.) durante condiciones de mercadeo. Proc. Interam. Soc. Trop. Hort. 41:209-214.

Balois-Morales R, M T Colinas L, C B Peña V, S H Chávez F, I Alia $T$ (2008) Sistema enzimático antisenescencia, catalasasuperoxido dismutasa de frutos de pitahaya (Hylocereus undatus) almacenados con frio. Rev. Chapingo S. Hort. 14:295-29920.

Bourne M (1980) Texture evaluation of horticultural crops. HortScience 15:51-57.

Centurión A R, S Solís, C Saucedo, R Báez, E Sauri (2008) Cambios físicos, químicos y sensoriales en frutos de pitahaya (Hylocereus undatus) durante su desarrollo. Rev. Fitotec. Mex. 31:1-5.

Centurión A R, S Solís, E Mercado, R Báez, C Saucedo, E Sauri (1999) Variación de las principales características de la pitahaya (Hylocereus undatus) durante su maduración postcosecha. Hort. Mex. 7:419-425.

Corrales G J (2003) Caracterización, Poscosecha, Aprovechamiento e Industrialización de Pitayas y Pitahayas. In: Pitayas y Pitahayas. C A Flores V (ed). Universidad Autónoma Chapingo, Chapingo Edo. de México. pp:137-173.

Díaz-Pérez J C (1998) Transpiration rates in eggplant fruit as affected by fruit and calyx size. Postharv. Biol. Technol. 13:45-49.

Gökmen V, N Kahraman, N Demir, J Acar (2000) Enzimatically validated liquid chromatographic method for the determination of ascorbic and dehydroascorbic acids in fruit and vegetables. J. Chromatography A.881:309-316.

Kader A A, M E Saltveit (2003) Respiration and Gas Exchange. In: Postharvest Physiology and Pathology of Vegetables. J A Bartz, J K Brecht (eds). 2nd ed. Marcel Dekker, Inc. New York, USA. pp:7-29.

Kader A A, N F Sommer, M L Arpaia (2002) Postharvest Handling Systems, Tropical Fruits. In: Postharvest Technology of Horticultural Crops. A A Kader (ed). University of California: Division of Agricultural and Natural Resources, USA. pp:385398.

Ladaniya M S (2008) Citrus Fruit. Biology, Technology and Evaluation. Academic Press, USA. 558 p.

Le Bellec F, F Vaillant, E Imbert (2006) Pitahaya (Hylocereus spp.): a new fruit crop, a market with a future. Fruits 61:237-250.

Meraz A, C Gómez, R Schwentesius (2003) Pitahaya de México, Producción y Comercialización en el Contexto Internacional.
In: Pitayas y Pitahayas. C A Flores V (ed). Universidad Autónoma Chapingo. pp:99-116.

Merten S (2003) A Review of Hylocereus production in the United States. J. Prof. Assoc. Cactus Develop. 1:98-105.

MINITAB (2004) Statistical software, Release number 14.0. Disponible en: www.minitab.com (septiembre 2009).

Minolta (1994) Precise Color Comunication. Color control from feeling to instrumentation. Minolta Co., Ltd. Osaka, Japan. 49 p.

Mizrahi Y, A Nerd, Y Sitrit (2002) New Fruits for Arid Climates. In: New Trends in New Crops and New Uses. J Janick, A Whipkey (eds). ASHS Press, Alexandria, VA. pp:378-384.

Nerd A, F Gutman, Y Mizrahi (1999) Ripening and postharvest behaviour of fruits of two Hylocereus undatus species (Cactaceae). Postharv. Biol. Technol. 17:39-45.

Osuna E T (2006) Validación de un huerto de pitahaya (Hylocereus undatus) en la zona centro de Sinaloa: fenología y determinación de madurez del fruto para cosecha. In: Memoria Anual, Ejercicio Operativo 2004-2005. Fundación Produce Sinaloa, A. C. Culiacán, Sinaloa, México pp:60-63.

Paliyath G, D P Murr (2008) Common Fruits, Vegetables, Flowers, and Their Quality Characteristics. In: Postharvest Biology and Technology of Fruits, Vegetables, and Flowers. G Paliyath, D P Murr, A K Handa, S. Lurie (eds). Wiley-Blackwell, USA. pp:8-18.

Phebe D, M K Chew, A A Suraini, O M Lai, O A Janna (2009) Redfleshed pitaya (Hylocereus polyrhizus) fruit colour and betacyanin content depend on maturity. Internatl. Res. J. 16:233-242.

Rodríguez D A, M del P Patiño, D Miranda, G Fischer, J A Galvis (2005) Efecto de dos índices de madurez y dos temperaturas de almacenamiento sobre el comportamiento en poscosecha de la pitahaya amarilla (Selenicereus megalanthus Haw.). Rev. Fac. Nac. Agron. Medellín 58:2837-2857.

SAGARPA, Secretaría de Agricultura, Ganadería, Desarrollo Rural, Pesca y Alimentación. (2009) Anuario Estadístico de la Producción Agrícola. Disponible en: http://www.siap.gob.mx (diciembre 2010).

Teixeira G H A, J F Durigan, M A Lima, R E Alves, H A C Filgueiras (2005) Postharvest changes and respiratory pattern of bacuri fruit (Platonia insignis Mart.) at different maturity stages during ambient storage. Acta Amaz. 35:17-21.

To L V, N Ngu, N D Duc, H T T Huong (2002) Dragon fruit quality and storage life: Effect of harvest time, use of plant growth regulators and modified atmosphere packaging. Acta Hort. 575:611-621

Wall M M, Khan S A (2008) Postharvest quality of dragon fruit (Hylocereus spp.) after X-ray irradiation quarantine treatment. HortScience 43:2115-2119.

Wills R, B McGlasson, D Graham, D Joyce (1998) Postharvest, An introduction to the Physiology and Handling of Fruit, Vegetables and Ornamentals. University of New South Wales. Press-Cab International. Sidney, Australia. 262 p. 